\title{
Factibilidad de la ventillación mecánica compartida
}

\section{Feasibility of shared mechanical ventilation}

\author{
Jorge Alberto Castañón-González, * Sergio Camacho-Juárez, Luis Antonio Gorordo-Delsol, \\ Jessica Garduño-López, Orlando Pérez-Nieto, Marco Antonio Amezcua-Gutiérrez y \\ Germán Fernández-de Alba Vejar \\ Secretaría de Salud, Hospital Juárez de México, Unidad de Cuidados Intensivos, Ciudad de México, México
}

Leímos con interés la carta al editor enviada por el doctor José Luis Sandoval Gutiérrez, en la que comenta nuestra publicación sobre ventilación mecánica simultánea con un solo ventilador a varios pacientes. ${ }^{1}$ En ella señala que debido a la variabilidad de la mecánica pulmonar y el estado de gravedad de los pacientes con síndrome de insuficiencia respiratoria aguda existirían complicaciones en el tratamiento; concluye que aún no es posible señalar que "donde se ventila uno, se ventilan dos."

Al respecto respondemos puntualmente:

1. En nuestro artículo claramente mencionamos que los candidatos deben tener procesos fisiopatológicos similares, por ejemplo, neumonía por COVID-19 y una presión de conducción similar, para lograr volúmenes corrientes aceptables y evitar ergotrauma.

2. Recientemente, en el Hospital Presbiteriano de Nueva York, el doctor Jeremy R. Beitler y su equipo llevaron a cabo con éxito la ventilación compartida durante 48 horas en tres pares de pacientes. La publicación se encuentra en prensa. ${ }^{2}$

3. Existen variaciones a esta técnica en la que se instalan al ventilador dos mezcladores (blenders) de oxígeno y válvulas para presión positiva al final de la espiración (PEEP) en cada ramal de los circuitos inspiratorios y espiratorios, respectivamente, para dosificar a cada paciente la fracción inspirada de oxígeno y PEEP. ${ }^{3}$ Si bien, como el mismo doctor Beitler señala en su artículo, "estas soluciones de ingeniería biomédica en presencia de un déficit de ventiladores y equipo, por la urgencia de las circunstancias, no son la mejor solución clínica en este contexto".

4. Afortunadamente, no hemos tenido la necesidad de aplicar esta técnica en México, pero podemos concluir que "donde se ventila un paciente, sí se pueden ventilar dos" en forma segura.

\section{Bibliografía}

1. Castañón-González JA, Camacho-Juárez S, Gorordo-Delsol LA, Garduño-López J, Pérez Nieto O, Amezcua-Gutiérrez MA, Fernández-de Alba Vejar G. Ventilación mecánica simultánea con un solo ventilador a varios pacientes. Gac Med Mex 2020;156:250-253.

2. Beitler JR, Mittel AM, Kallet R, Kacmarek R, Hess D, et al. Ventilator sharing during an acute shortage caused by COVID-19 pandemic. Am J Respir Crit Care Med. 2020 Jun 9. DOI: 10.1164/rccm.202005-1586LE

3. Han JS, Mashari A, Singh D, Dianti J, Goliher E, Long M, et al. Personalized ventilation to multiple patients using a single ventilator: Description and proof of concept. Crit Care Explor 2020:2(5);e0118. DOI: 10.1097/CCE.0000000000000118

\section{Correspondencia:}

*Jorge Alberto Castañón-González

E-mail: jorge.castanong@gmail.com

0016-3813/@ 2020 Academia Nacional de Medicina de México, A.C. Publicado por Permanyer. Este es un artículo open access bajo la licencia CC BY-NC-ND (http://creativecommons.org/licenses/by-nc-nd/4.0/).
Gac Med Mex. 2019;155:368-368

Disponible en PubMed

www.gacetamedicademexico.com 